\title{
EL APEGO ADULTO Y LA CALIDAD PERCIBIDA DE LAS \\ RELACIONES DE PAREJA: EVIDENCIAS A PARTIR DE UNA POBLACIÓN ADULTA JOVEN
}

ADULT ATTACHMENT AND THE PERCEIVED QUALITY OF RELATIONSHIPS: EVIDENCE FROM A YOUNG ADULT POPULATION

Recibido: 20 de 0ctubre de 2016 | Aceptado: 25 de Noviembre de 2016

DOI: 10.22199/507187475.2016.0003.00005

\author{
CAMILA J. MEDINA 1 ; LAURA Y. RIVERA 1 ; JOSÉ A. AGUASVIVAS 1 \\ 1. UNIVERSIDAD IBEROAMERICANA, Santo Domingo, República Dominicana
}

\begin{abstract}
RESUMEN
INTRODUCCIÓN: Diversos estudios resaltan la importancia de las relaciones de pareja en el desarrollo psicosocial y la salud física y emocional de las personas. Adicionalmente, se ha encontrado que los tipos de apego tienen una capacidad predictiva sobre la calidad percibida de las relaciones de pareja. Sin embargo, la información que se tiene al respecto para la población adulta joven es limitada. OBJETIVO: evaluar la correlación entre los estilos de apego y diferentes componentes de las relaciones de pareja en una población adulta joven. INSTRUMENTOS: Los participantes respondieron al cuestionario Experience in Close Relationships (32 ítems) y al Perceived Relationship Quality Components (18 ítems). MÉTOD0: La muestra estuvo compuesta por 119 estudiantes universitarios dominicanos de ambos sexos y edades entre 18 y 26 años (M $=20.03$; DT $=1.95$ ). RESULTADOS: se encontró un patrón de correlacional distinto para los tipos de apego, teniéndose que el Apego Ansioso no correlacionó con la mayoría de los componentes de calidad percibida, mientras que para el apego evitativo las correlaciones fueron negativas y altas con las escalas de Satisfacción, Compromiso, Intimidad, Amor y Calidad General. Adicionalmente, los hombres tuvieron medias significativamente más altas de Apego Evitativo. CONCLUSIONES: Los hallazgos ilustran la importancia de los estilos de apego, en particular del Apego Evitativo, respecto a la calidad de las relaciones de pareja en la adultez temprana.
\end{abstract}

PALABRAS CLAVE: Apego evitativo, apego ansioso, relación de pareja, calidad percibida.

\section{ABSTRACT}

INTRODUCTION: Many studies highlight the importance of relationships during people's psychological development as well as their physical and emotional health. These studies also show that the types of attachment have a predictable capacity regarding the perceived quality in relationships. However, few have been said about the topic in young adult population. OBJECTIVE: To evaluate the correlation between attachment styles and different components of relationships in young adult population. METHOD: The sample was composed of 119 Dominican university students whose ages ranged between 18 and 26 ( $M=20.03$; DT = 1.95). RESULTS: There is a different correlational pattern for type of attachment. Anxious-Ambivalent Attachment did not correlate with most components of perceived quality; whereas Anxious-Avoidant Attachment correlated negatively and high in the scales of satisfaction, commitment, intimacy, love, and general quality. Additionally, men registered meaningfully higher means of Anxious-Avoidant Attachment. CONCLUSIONS: These findings illustrate the importance of the styles of attachment, specially the Anxious-Avoidant Attachment regarding the quality of relationships in young adults.

KEY WORDS: Anxious-Avoidant Attachment, Anxious-Ambivalent Attachment, relationships, perceived quality.

1. Para correspondencias en relación a este artículo pueden ser dirigidas a Camila Medina, Escuela de Psicología, Universidad Iberoamericana. Ave. Francia No. 129, Gazcue, Santo Domingo, República Dominicana. E-mail: camilajmedina@gmail.com 
La salud física y emocional, así como el desarrollo psicosocial de las personas, están estrechamente enlazados con sus relaciones afectivas e íntimas (Guzmán \& Contreras, 2012; Martínez, Fuertes, Orgaz, Vicario \& González, 2014). Debido a esto, diversos investigadores y profesionales del área de la salud se han enfocado en lo concerniente a las dinámicas de las relaciones de pareja y las posibles variables generadoras de conflictos (e.g., Gottman \& Levenson, 1999; Guzmán \& Contreras, 2012; Karney \& Bradbury, 1995). Así, se ha encontrado que mientras para algunas personas los conflictos con la pareja son esporádicos y ocurren mayormente bajo situaciones estresantes, para otras estos episodios suceden regularmente durante las interacciones del día a día, dificultándoles la estabilidad de sus relaciones y ocasionando rupturas y divorcios (Amato, 2000; Campbell, Boldry, Simpson \& Kashy, 2005). Más aún, las personas involucradas en conflictos de pareja recurrentes tienden a experimentar mayores niveles de depresión, baja satisfacción y más problemas de salud que individuos en relaciones de pareja estables (Amato, 2000).

Debido a la complejidad de las relaciones de pareja, las causas y consecuencias de los conflictos pueden ser visualizados desde diferentes perspectivas (Amato \& Previti, 2003). Entre éstas se encuentra la perspectiva sociológica, cuyos hallazgos se enfocan en causas de conflicto tales como estructura y ciclo de vida marital, destacando la clase social, la etnia y la edad como elementos importantes en las relaciones de pareja (Bumpass, Martin \& Sweet, 1991; White, 1990). Otro enfoque es el psicológico, donde los elementos detonantes de conflicto en las interacciones de pareja son las características de la personalidad y la capacidad de resolución de problemas (Amato \& Previti, 2003; Leonard \& Roberts, 1998). relación estable y madura, que son: (1) el compromiso, el cual se refiere al interés y la responsabilidad hacia la relación; (2) la intimidad, que es la capacidad de establecer cercanía afectiva; (3) el romance, referente a las conductas establecidas socialmente para generar atracción; y (4) el amor, que es un proceso funcional que ayuda a establecer relaciones íntimas. Afirma, asimismo, que mientras los tres primeros componentes son de tipo social y dependen de la cultura en donde se vive, el amor es biológico e innato, independiente de la cultura y específico en cada individuo. Además, la interacción y buen funcionamiento de cada uno de estos componentes es imprescindible para la calidad de la relación, definiéndose ésta como el grado en que los miembros de la pareja son capaces de intimar y brindarse afecto y apoyo mutuo (Collins, Welsh \& Furman, 2009; Martínez et al., 2014).

Una percepción elevada de la calidad de la relación se asocia frecuentemente con altos niveles de bienestar, satisfacción y compromiso en la pareja, y un mejor pronóstico en relaciones posteriores (Collins et al., 2009; Martínez et al., 2014). Además, la calidad percibida de la relación por sus miembros guarda una estrecha vinculación con los patrones relacionales que estos desarrollaron en su infancia (Martínez et al., 2014). Mientras mejores sean las características del ambiente familiar, en cuanto a la calidez parental y estabilidad emocional, y exista una convivencia afectiva por parte de los miembros de la pareja, mayor será la capacidad de experimentar una relación amorosa estable y satisfactoria (Auslander, Short, Sucop \& Rosenthal, 2009). Este hallazgo ha sido corroborado por numerosas investigaciones, destacándose la teoría del apego como uno de los referentes más relevantes (e.g., Hazan \& Shaver, 1987; Martínez et al., 2014; Roisman et al., 2007; Simpson, 1990).

Maureira (2011) destaca cuatro componentes fundamentales para una 
Teoría del Apego

En las últimas dos décadas, la teoría del apego (Bowlby, 1969, 1973, 1980) se ha situado como uno de los marcos conceptuales más relevantes a la hora de comprender el proceso de regulación afectiva de las relaciones (Duemmeler \& Kobak, 2001; Mikulincer, Shaver, \& Pereg, 2003). Esta teoría se refiere al lazo que desarrolla el infante hacia el cuidador primario, usualmente la madre, y a las consecuencias que este vínculo conlleva en la formación de su autoconcepto y en el desarrollo de su percepción sobre su universo social (Collins \& Read, 1990; Yárnoz, Alonoso-Arbiol, Plazaola \& Sainz, 2001). La propuesta de Bowlby, conocida como el primer enfoque formal y evolutivo sobre el apego, establece que la relación de apego tiene un profundo impacto en la estructura de la personalidad del niño. Asimismo, la misma postula que el desarrollo del apego está estrechamente determinado por las habilidades emocionales y la capacidad de respuesta del cuidador ante las necesidades del infante (Bowlby 1973, 1980; Hazan \& Shaver, 1987).

Dependiendo de cómo haya sido la relación del niño con su cuidador primario, el apego puede clasificarse en tres tipos (Ortiz, Gómez \& Apocada, 2002): seguro, inseguro evitativo e inseguro ansioso 0 ambivalente. El primer tipo, el apego seguro, suele manifestarse en individuos que recuerdan una relación cariñosa con madres sensibles y preocupadas por ellos. El segundo tipo, el apego inseguro evitativo, se presenta en quienes generalmente cuentan una historia fría, ya que su figura de apego manifestó sentimientos de rechazo y careció de capacidad para responder a sus necesidades tempranas. El tercer tipo es el apego inseguro ansioso, que lo exhiben quienes recuerdan un tono afectivo negativo entre los padres y una relación con una figura de apego poco comprensiva (Ortiz et al., 2002).
A pesar de que los estilos de apego se asumen como universales, las conductas generadas por estos presentan diferencias individuales que pueden ser explicadas por los modelos operativos internos (MOl; Guzmán \& Contreras, 2012). Los MOI son esquemas relacionales relativamente estables a través de los cuales un individuo se hace de sí mismo (self) y de otros en un contexto social (Feeney \& Collins, 2001; Yárnoz et al., 2001). A partir de este enfoque junto con los tipos de apego se distinguen cuatro perspectivas: segura, con una idea positiva de sí mismo y los demás; evitativa-rechazante, con una idea positiva de sí mismo e idea negativa de los demás; preocupado, idea negativa de sí mismo y positiva de los demás; y evitativo-temeroso, en donde la idea del sí mismo, así como la de los demás es negativa (Guzmán \& Contreras, 2012; Yárnoz et al., 2001).

Debido a que la formación de las relaciones cercanas con otras personas se describe como un proceso homólogo al del desarrollo del apego (Fraley \& Davis, 1997), las personas tienden a desarrollar diferentes estilos de relacionarse en la adultez, determinando así la forma en que los individuos perciben e interaccionan con personas importantes en su entorno (Griffin \& Bartholomew, 1994). De manera particular, el apego juega un papel especialmente importante en las relaciones románticas (Tucker \& Anders, 1999).

Importancia del Apego en las Relaciones de Pareja

Las experiencias interpersonales durante la infancia juegan un rol importante en la seguridad del apego que se desarrolla en las relaciones de pareja en la adultez (Feldman, Gowen, \& Fisher, 1998; Hazan \& Shaver, 1987). Este estilo de apego adulto, que se empieza a manifestar a partir de la adolescencia, ha sido numerosas veces asociado a aspectos como la estabilidad de la relación, la capacidad de resolución de problemas de la pareja y la satisfacción y calidad percibida de la relación (e.g., 
Auslander et al., 2009; Heffernan, Fraly, Vicary, \& Brumbaug, 2012; Mikulincer, et al., 2003; Molero, Shaver, Ferrer, Cuadrado, \& Alonso-Arbiol, 2010).

En general, los individuos con un estilo de apego seguro experimentan un nivel más elevado de satisfacción en sus relaciones íntimas, mostrando confianza, apertura para ofrecer y recibir apoyo y capacidad para la resolución de situaciones cotidianas, logrando un mayor disfrute de la relación (Brennan \& Shaver, 1995; Feeney \& Noller, 1990; Hazan \& Shaver, 1987; Ortiz et al., 2002). En cambio las personas con un estilo de apego inseguro evitativo se les dificulta mantener relaciones interpersonales, debido a que no logran manejar los niveles de intimidad y confianza necesarios en una relación de pareja, manifestando una lejanía afectiva que les impide comprometerse (Gómez-Zapiain, Ortiz \& Gómez-Lope, 2012; Ortiz, et al., 2002). Por último, los que presentan un estilo de apego inseguro ansioso tienden a experimentar una frecuente sensación de abandono y desconfianza con su pareja, manifestando una constante búsqueda de afecto $e$ intimidad caracterizadas por la inseguridad (Brennan \& Shaver, 1995; Feeney \& Noller, 1990; Hazan \& Shaver, 1987; Ortiz et al., 2002).

Si bien el estilo de apego adulto y su influencia sobre el matrimonio ha sido ampliamente estudiado (e.g., Amato \& Previti, 2003; Gottman \& Levenson, 1999; Hazan \& Shazer, 1987; Kachadourian, Fincham, \& Davila, 2004), no son abundantes los estudios que directamente se han enfocado en las relaciones de pareja en la población de adultos jóvenes (Duemmler \& Kobak, 2001; Rivera, Ruz \& Muñoz, 2011). Los hallazgos con que se cuenta actualmente sobre esta población apuntan a que durante esta etapa las personas con apego seguro tienden a reportar relaciones más felices, amistosas y de confianza, teniendo éstas una mayor duración. En cambio, dentro del apego inseguro, los de tipo evitativo reportan temor a la cercanía y frecuentes altibajos emocionales, con relaciones de duración variable; y los de tipo ansioso reportan relaciones caracterizadas por los celos, la obsesión o la atracción sexual extrema (Duemmler \& Kobak, 2001; Feeney \& Collins, 2001; Guzmán \& Contreras, 2012; Martínez et al., 2014).

Por otro lado, los estudios en los que se ha tomado en consideración posibles diferencias de género en los estilos de apego adulto en su mayoría destacan que, tanto el apego inseguro evitativo como el ansioso, son predictores de la insatisfacción en las relaciones de pareja de las mujeres, mientras que, para los hombres, sólo el apego evitativo predice la insatisfacción (Brennan \& Shaver, 1995; Kirkpatrick \& Davis, 1994; Mikulincer \& Shaver, 2007). Kirkpatrick y Davis (1994) explican estas diferencias a través de los roles de género, enfatizando que, como las mujeres por lo general realizan más esfuerzos para el mantenimiento y ruptura de las relaciones, su estilo de apego ejerce una influencia mayor sobre la satisfacción en la relación que el estilo de apego del hombre. Además, sostienen que como los hombres ansiosos por lo general se emparejan con mujeres con apego ansioso o seguro, en el largo plazo, las relaciones tienden a ser estables y aparentemente satisfactorias (Kirkpatrick \& Davis, 1994).

\section{Objetivos del presente estudio}

Como se ha visto anteriormente, dependiendo de las características de la relación que se tuvo con los cuidadores primarios durante la infancia, las personas desarrollan diferentes tipos de apego que luego pueden incidir en su estilo de relación afectiva en la adultez. Esto se conoce como el estilo de apego adulto e influye sobre la satisfacción y la calidad percibida de las relaciones en sus diferentes componentes, tales como el compromiso, la intimidad, la confianza y el amor. El presente estudio 
tiene como objetivo, por lo tanto, evaluar la posible correlación entre los estilos de apego inseguros y los componentes de la relación de pareja en jóvenes adultos, así como las diferencias de género que pudieran existir en dichas correlaciones. Basado en lo expuesto previamente, se han formulado las siguientes hipótesis:

Hipótesis 1: se esperan correlaciones negativas entre el estilo de apego evitativo y los componentes de calidad de la relación de pareja.

Hipótesis 2: se esperan correlaciones negativas entre el estilo de apego ansioso y los componentes de calidad de la relación de pareja.

\section{MÉTODO}

\section{Participantes}

El presente estudio se llevó a cabo con estudiantes de una universidad privada en Santo Domingo, República Dominicana. En base a un muestreo por conveniencia se obtuvo una muestra de 119 adultos jóvenes universitarios de ambos sexos, de los cuales 69 fueron mujeres y 50 hombres, y cuyas edades estaban comprendidas entre los 18 y los 26 años (Media = 20.03; desviación típica $=1.95)$. Adicionalmente, un $95.0 \%$ de los individuos se encontraban en una relación de noviazgo, un $3.3 \%$ en unión libre y un $1.7 \%$ estaban casados. Por otro lado, los participantes estudiaban las siguientes carreras: Medicina (26.9\%), Mercadeo (16.8\%), Administración de Empresas (13.4\%), Psicología (12.6\%), Ingeniería Industrial (7.6\%), Ingeniería Civil (6.7\%), Ingeniería en Tecnologías de la Información y Comunicación (5.0\%), Odontología (5.0\%), Derecho (4.2\%), Arquitectura $(0.9 \%)$ y Diseño de Interiores (0.9\%). Asimismo, el $80.6 \%$ de la muestra era procedente de la República Dominicana, el $10.1 \%$ de los Estados Unidos de América, el $3.4 \%$ de Puerto Rico, el $0.9 \%$ de Haití y el $5.0 \%$ restante no informó su nacionalidad.

\section{Instrumentos}

Perceived Relationship Quality Components (PRQC; Fletcher, Simpson \& Thomas, 2000). EI PRQC explora la forma en que los individuos perciben la calidad de las relaciones de pareja y está compuesto por las sub-escalas de Satisfacción, Compromiso, Intimidad, Confianza, Pasión y Amor, y por una escala global que mide la Calidad General de la relación. El PRQC cuenta con un total de 18 ítems, los cuales están distribuidos equitativamente en las 6 sub-escalas (3 por cada sub-escala). Los autores del instrumento reportan niveles de consistencia interna entre adecuados y excelentes para las diferentes escalas (alfa de Cronbach desde 0.78 para Confianza hasta 0.96 para Compromiso). Cada ítem fue evaluado a partir de una escala de respuesta tipo Likert de 5 puntos, donde las opciones eran "nada" (1), "poco" (2), "más o menos" (3), "mucho" (4) y "completamente" (5). Los enunciados en español del PRQC se muestran en el Apéndice.

Experience in Close Relationships (ECR; Brennan, Clark, \& Shaver, 1998). Esta medida fue utilizada para la valoración del apego adulto a partir de su adaptación española (Alonso-Arbiol, Balluerka \& Shaver, 2007). El ECR está compuesto por las sub-escalas de Apego Ansioso, con 15 ítems (e.g., "Me preocupa bastante el hecho de perder a mi pareja"), y de Apego Evitativo, con 17 ítems (e.g., "Me siento irritado/a cuando mi pareja quiere demasiada intimidad afectiva"). Ambas escalas mostraron fiabilidades buenas en su versión española (alfa de Cronbach de 0.82 para el Ansioso y de 0.87 para el Evitativo). Los ítems se evaluaron a partir de una escala Likert de 5 puntos, con las opciones "nada de acuerdo" (1), "poco de acuerdo" (2), "más o menos de acuerdo" (3), "muy de acuerdo" (4), y "totalmente de acuerdo" (5).

Ítems Dirigidos de Control de la Atención. Siguiendo las recomendaciones de Maniaci y Rogge (2014), se incluyeron 
en la batería 3 ítems de control de la atención (e.g., "Si se encuentra leyendo esta pregunta por favor marcar la opción poco de acuerdo"). Aquellos participantes que respondieron incorrectamente a dos 0 a los tres ítems dirigidos fueron eliminados de la muestra.

\section{Procedimiento}

Antes del inicio del estudio se obtuvo la autorización del comité de ética de la institución educativa. Posteriormente, se obtuvo la autorización de la facultad y se procedió a contactar los estudiantes en sus respectivas aulas, lugar donde se les explicó la naturaleza del estudio y se les invitó a participar, suministrándoles un consentimiento informado. Con los estudiantes que accedieron, se procedió a la administración de la batería de forma anónima, dándoles de forma paralela las instrucciones de lugar. Seguidamente, los estudiantes procedieron a completar los cuestionarios durante su período regular de clases, con una media de tiempo de 15 minutos. En total respondieron 120 estudiantes a la batería, mas uno de ellos tuvo que ser eliminado por no responder adecuadamente a los ítems de control de la atención, dejando la muestra final en 119 participantes.

Los ítems del PRQC fueron traducidos del inglés al español para el presente estudio por dos personas con nivel fluido en ambos idiomas. Para el proceso de traducción se utilizó la técnica paralelociega. En primer lugar, las dos personas hicieron traducciones por separado desde la fuente (inglés) a la lengua objetivo (español). En segundo lugar, las dos traducciones fueron comparadas y las discrepancias se resolvieron para llegar a las versiones óptimas de los ítems. Finalmente, los ítems fueron testeados en un estudio piloto para comprobar su correcta comprensión.

\section{Análisis Estadísticos}

Para el manejo y análisis de los datos se utilizó el software IBM SPSS Statistics Versión 20 (IBM Corporation 1989-2011). Para calcular la fiabilidad se utilizó el coeficiente alfa de Cronbach (a), el cual se interpretó según la guía proporcionada por George y Mallery (2003): $a \geq 0.90$ se considera excelente, $a \geq 0.80$ y $<0.90$ se considera buena, $a \geq 0.70$ y $<0.80$ se considera aceptable, $a \geq 0.60$ y $<0.70$ se considera cuestionable, $a \geq 0.50$ y $<0.60$ se considera pobre, y un $a<0.50$ se considera inaceptable. Asimismo, para contrastar las hipótesis planteadas y evaluar el grado de relación entre las diferentes escalas se utilizó el coeficiente de correlación de Pearson (r). Según Cohen (1992), valores de $|r| \geq 0.50$ son indicativos de correlaciones altas, $|r| \geq 0.30 \mathrm{y}<0.50$ de correlaciones medianas y $|r| \geq 0.10$ y $<0.30$ de correlaciones bajas. Por otro lado, para comparar la magnitud de las correlaciones entre hombres y mujeres se utilizó la transformación de $r$ a $Z$ de Fisher. Finalmente, se utilizó la prueba $T$ de Student para muestras independientes para comparar las puntuaciones medias en las escalas de apego entre hombres y mujeres. Los tamaños del efecto de las diferencias fueron evaluados a partir de la $d$ de Cohen, donde valores de $|\mathrm{d}| \geq 0.80$ indican efectos grandes, $|\mathrm{d}| \geq 0.50 \mathrm{y}<0.80$ efectos medianos y $|\mathrm{d}| \geq 0.20$ y $<0.50$ denotan efectos pequeños (Cohen, 1992).

\section{RESULTADOS}

Análisis Descriptivos y Fiabilidades de las Escalas

En la Tabla 1 se muestran los estadísticos descriptivos para las escalas del ECR y el $P R Q C$. Respecto a las fiabilidades de las escalas, tanto la de Apego Ansioso como la de Apego Evitativo alcanzaron una fiabilidad de 0.81, considerada como buena. En el caso de los componentes de calidad percibida de las relaciones, la escala de Satisfacción alcanzó una fiabilidad 
excelente $(\alpha=0.92)$, las escalas General ( $\alpha$ $=0.89)$, de Confianza $(a=0.84)$ y de Compromiso ( $a=0.83$ ) una fiabilidad buena, las de Intimidad $(a=0.73)$ y Amor ( $a$ $=0.73$ ) una fiabilidad aceptable, y la de Pasión (0.61), una fiabilidad cuestionable.
Como se puede ver, todas las escalas mostraron un nivel de fiabilidad adecuado 0 mejor, con excepción de la Pasión, cuyos resultados se deberán interpretar con cautela.

TABLA 1.

Estadísticos Descriptivos para las Escalas del ECR y el PRQC.

\begin{tabular}{|c|c|c|c|c|c|c|c|}
\hline \multirow[b]{2}{*}{ Test/Escala } & \multirow[b]{2}{*}{ \# Ítems } & \multicolumn{3}{|c|}{ Media } & \multicolumn{3}{|c|}{ Desviación Tipica } \\
\hline & & M & $\mathrm{H}$ & T & M & $\mathrm{H}$ & T \\
\hline \multicolumn{8}{|l|}{$E C R$} \\
\hline Ansioso & 15 & 37.72 & 38.46 & 38.03 & 8.48 & 11.36 & 9.75 \\
\hline Evitativo & 17 & 34.29 & 38.02 & 35.86 & 9.14 & 9.55 & 9.45 \\
\hline \multicolumn{8}{|l|}{$P R Q C$} \\
\hline Satisfacción & 3 & 13.16 & 12.50 & 12.88 & 1.91 & 2.38 & 2.14 \\
\hline Compromiso & 3 & 13.10 & 12.36 & 12.79 & 1.74 & 2.41 & 2.07 \\
\hline Intimidad & 3 & 13.54 & 13.02 & 13.32 & 1.48 & 2.14 & 1.80 \\
\hline Confianza & 3 & 13.46 & 13.48 & 13.47 & 1.76 & 2.07 & 1.89 \\
\hline Pasión & 3 & 11.71 & 11.72 & 11.71 & 2.20 & 2.52 & 2.33 \\
\hline Amor & 3 & 13.70 & 13.02 & 13.41 & 1.39 & 2.25 & 1.82 \\
\hline General & 18 & 78.67 & 76.10 & 77.59 & 6.96 & 10.47 & 8.66 \\
\hline
\end{tabular}

Nota. $\mathrm{M}=$ mujeres; $\mathrm{H}=$ hombres; $\mathrm{T}=$ total; $\mathrm{ECR}=$ Experience in Close Relationships; $\mathrm{PRQC}=$ Perceived Relationship Quality Components; $n=69$ para las mujeres y $n=50$ para los hombres.

Correlaciones entre el Apego y la Calidad de la Relación de Pareja

En la Tabla 2 se muestran las correlaciones de Pearson entre las escalas de apego y de calidad de la relación de pareja, primero por separado para mujeres y hombres, y luego para la muestra total. Es importante destacar que sólo dos correlaciones mostraron diferencias estadísticamente significativas $(p<0.05)$ entre mujeres y hombres: los hombres mostraron correlaciones más altas entre Intimidad y Pasión ( $r=0.72$ ) e Intimidad y Calidad General $(r=0.88)$ que las mujeres, para las cuales dichas correlaciones fueron de $0.31 \mathrm{y}$ 0.76 , respectivamente.

Como se muestra en la Tabla 2, las puntuaciones de Apego Evitativo en general correlacionaron de manera negativa alta con las de calidad de la relación de pareja. Las correlaciones negativas más altas se obtuvieron para las escalas de Calidad General ( $r=-0.63)$, Compromiso $(r=-0.57)$, Intimidad $(r=-0.51)$, Amor $(r=-0.51)$ y Satisfacción $(r=-0.50)$. Estos resultados apoyan la Hipótesis 1 del estudio. A diferencia de estos resultados, las puntuaciones de Apego Ansioso sólo correlacionaron de manera significativa con las escalas de Satisfacción $(r=-0.27, p<$ $0.01)$ y Compromiso $(r=-0.19, p<0.05)$. En este caso, los resultados sólo apoyan parcialmente la Hipótesis 2, y evidencian la mayor relevancia del Apego Evitativo en cuanto a la calidad de las relaciones de pareja. 
TABLA 2.

Correlaciones entre las Escalas del ECR y el PRQC.

\begin{tabular}{|c|c|c|c|c|c|c|c|c|c|}
\hline Grupo/Escala & ECR1 & ECR2 & RQC1 & RQC2 & RQC3 & RQC4 & RQC5 & RQC6 & RQC7 \\
\hline \multicolumn{10}{|l|}{ Mujeres ${ }^{a}$} \\
\hline ECR1. Ansioso & 1.00 & & & & & & & & \\
\hline ECR2. Evitativo & 0.06 & 1.00 & & & & & & & \\
\hline PRQC1. Satisfacción & $-0.41^{* *}$ & $-0.43^{* *}$ & 1.00 & & & & & & \\
\hline PRQC2. Compromiso & $-0.29^{*}$ & $-0.46^{* *}$ & $0.72^{* \star}$ & 1.00 & & & & & \\
\hline PRQC3. Intimidad & -0.05 & $-0.52^{* *}$ & $0.64^{* *}$ & $0.45^{* *}$ & 1.00 & & & & \\
\hline PRQC4. Confianza & -0.11 & $-0.29^{*}$ & $0.37^{\star *}$ & 0.19 & $0.37^{* *}$ & 1.00 & & & \\
\hline PRQC5. Pasión & 0.07 & $-0.30^{*}$ & 0.19 & 0.11 & $\underline{0.31^{* *}}$ & -0.06 & 1.00 & & \\
\hline PRQC6. Amor & -0.05 & $-0.41^{* *}$ & $0.51^{* *}$ & $0.56^{* *}$ & $\overline{0.35} 5^{* *}$ & $0.39^{* *}$ & 0.11 & 1.00 & \\
\hline PRQC7. General & -0.21 & $-0.59^{* *}$ & $0.85^{* *}$ & $0.74^{* *}$ & $\underline{0.76}^{* *}$ & $0.54^{* *}$ & $0.47^{* *}$ & $0.69^{* *}$ & 1.00 \\
\hline \multicolumn{10}{|l|}{ Hombresa } \\
\hline ECR1. Ansioso & 1.00 & & & & & & & & \\
\hline ECR2. Evitativo & 0.13 & 1.00 & & & & & & & \\
\hline PRQC1. Satisfacción & -0.15 & $-0.55^{\star *}$ & 1.00 & & & & & & \\
\hline PRQC2. Compromiso & -0.11 & $-0.67^{* *}$ & $0.79^{* \star}$ & 1.00 & & & & & \\
\hline PRQC3. Intimidad & -0.24 & $-0.49^{* *}$ & $0.72^{\star *}$ & $0.53^{* *}$ & 1.00 & & & & \\
\hline PRQC4. Confianza & -0.22 & $-0.53^{\star *}$ & $0.55^{\star *}$ & $0.51^{* *}$ & $0.57^{* *}$ & 1.00 & & & \\
\hline PRQC5. Pasión & -0.10 & -0.19 & 0.27 & 0.08 & $\underline{0.72}^{* *}$ & $0.30^{*}$ & 1.00 & & \\
\hline PRQC6. Amor & 0.16 & $-0.58^{* *}$ & $0.60^{* *}$ & $0.72^{* *}$ & $\overline{0.48^{* *}}$ & $0.46^{* *}$ & 0.19 & 1.00 & \\
\hline PRQC7. General & -0.14 & $-0.65^{* *}$ & $0.86^{* *}$ & $0.79^{* *}$ & $\underline{0.88^{* *}}$ & $0.73^{* *}$ & $0.57^{* \star}$ & $0.75^{* \star}$ & 1.00 \\
\hline \multicolumn{10}{|l|}{ Total } \\
\hline ECR1. Ansioso & 1.00 & & & & & & & & \\
\hline ECR2. Evitativo & 0.10 & 1.00 & & & & & & & \\
\hline PRQC1. Satisfacción & $-0.27^{* *}$ & $-0.50^{* *}$ & 1.00 & & & & & & \\
\hline PRQC2. Compromiso & $-0.19^{*}$ & $-0.57^{\star *}$ & $0.77^{\star *}$ & 1.00 & & & & & \\
\hline PRQC3. Intimidad & -0.17 & $-0.51^{* *}$ & $0.69^{\star *}$ & $0.51^{* *}$ & 1.00 & & & & \\
\hline PRQC4. Confianza & -0.17 & $-0.39^{* *}$ & $0.46^{* *}$ & $0.36^{* *}$ & $0.47^{* *}$ & 1.00 & & & \\
\hline PRQC5. Pasión & -0.02 & $-0.25^{\star *}$ & $0.23^{*}$ & 0.09 & $0.53^{* *}$ & 0.12 & 1.00 & & \\
\hline PRQC6. Amor & 0.07 & $-0.51^{* *}$ & $0.58^{\star *}$ & $0.67^{* *}$ & $0.45^{* *}$ & $0.42^{* *}$ & 0.15 & 1.00 & \\
\hline PRQC7. General & -0.18 & $-0.63^{\star *}$ & $0.85^{* *}$ & $0.78^{* *}$ & $0.84^{* *}$ & $0.63^{* *}$ & $0.51^{* *}$ & $0.74^{* *}$ & 1.00 \\
\hline
\end{tabular}

Nota. ECR = Experience in Close Relationships; $\mathrm{PRQC}=$ Perceived Relationship Quality Components.

aLas correlaciones que son significativamente distintas $(p<0.05)$ entre mujeres y hombres aparecen subrayadas.

${ }^{*} p<0.05 ;{ }^{* *} p<0.01$.

Comparación de las Puntuaciones Medias por Género

Para comparar las puntuaciones de mujeres y hombres en las escalas de apego y calidad de la relación de pareja se utilizó la Prueba $\mathrm{T}$ de Student. Los resultados de estos análisis reflejaron diferencias estadísticamente significativas únicamente para la escala de Apego Evitativo, para la cual asumiendo varianzas iguales $(p=0.79)$ los hombres mostraron puntuaciones medias $(\mathrm{M}=38.02$, $\mathrm{DT}=9.548)$ más altas que las mujeres $(M=34.29$, $D T=9.135)$, con un efecto pequeño de acuerdo con los criterios de Cohen $(t[177]=2.157, p=0.33$, $d=0.40$ ). No obstante, es preciso tomar en cuenta que es posible que no se hayan detectado otras diferencias significativas entre escalas debido a una falta de potencia producto del tamaño muestral pequeño por grupo. 
DISCUSIÓN

Diversos estudios han evaluado la importancia del apego adulto en la calidad percibida de las relaciones de pareja, considerando como relevante su correlación (e.g., Feeney \& Collins, 2001; Hazan \& Shaver, 1987; Mikulincer, Florian, Cowan, \& Cowan, 2002). Sin embargo, son escasos aquellos que se han contextualizado en la adultez temprana 0 que han evaluado las diferencias de género entre dichas relaciones (Brennan \& Shaver, 1995; Duemmler \& Kobak, 2001; Kirkpatrick \& Davis, 1994; Tucker \& Anders, 1999). Por esta razón, el objetivo del presente estudio fue evaluar la relación entre los estilos de apego adulto y los diferentes componentes de las relaciones de pareja en una población de adultos jóvenes.

Con respecto al Apego Evitativo, éste correlacionó de manera negativa con todas las dimensiones de la relación de pareja. Las correlaciones de este tipo de apego fueron altas con las dimensiones de Compromiso, Satisfacción, Amor e Intimidad, mediana con la Confianza y baja con la Pasión. Además, correlacionó de manera alta y negativa con la dimensión de Calidad General, evidenciándose así su gran relevancia con respecto al funcionamiento global de las relaciones de pareja. Estos resultados son congruentes con la literatura, que sostiene que las personas con este tipo de apego tienden a considerarse autosuficientes, restándole importancia a las relaciones afectivas, mostrando dificultad en manejar los niveles de compromiso y confianza fundamentales en una relación de pareja, lo que repercute sobre la calidad percibida de la misma (Brennan \& Shaver, 1995; Feeney \& Noller, 1990; Hazan \& Shaver, 1987; Ortiz, et al., 2002, Martínez et al., 2014). Adicionalmente, estos resultados parecen ser congruentes con lo planteado por Mikulincer y Shaver (2007), quienes afirman que es posible que las conductas de evitación en los hombres sean especialmente desagradables para las mujeres, lo que se traduce en constantes quejas y conflictos que directamente afectan la calidad percibida de la relación para el hombre.

En cuanto al Apego Ansioso, las correlaciones fueron bajas y negativas con las variables de Satisfacción y Compromiso, mas no fueron significativas con las demás variables de la relación de pareja, ni con la Calidad General, lo que indica que no posee tanta relevancia en relación al funcionamiento de las relaciones de pareja. La literatura sostiene que las personas con este tipo de apego constantemente buscan intimidad en las relaciones, recurriendo a conductas preocupadas, dependientes y un tanto obsesivas para obtener la atención del otro y cubrir sus necesidades afectivas (Brennan \& Shaver, 1995; Collins \& Feeney, 2000; Gómez et al., 2011). No obstante, parece que estas conductas son menos negativas especto a la calidad percibida de las relaciones que aquellas que tipifican el Apego Evitativo (Gómez-Zapiain et al., 2012).

A pesar de que la forma de experimentar la satisfacción con la relación no varía dependiendo de si se es hombre o mujer (Guzman \& Contreras, 2012), se han encontrado ciertas diferencias de género en torno a la correlación entre el apego y los componentes de la relación. Para el estudio actual, en primer lugar, los resultados reflejan que el Apego Evitativo correlaciona en igual medida para hombres y mujeres con los componentes de la relación de pareja. No obstante, el Apego Ansioso se encuentra ligeramente relacionado con algunos componentes de la relación en el grupo femenino, pero no en el masculino. En segundo lugar, los hombres muestran puntuaciones más altas que las mujeres en el Apego Evitativo, lo que refleja en ellos una mayor tendencia hacia los comportamientos indicativos de este tipo de apego.

Los resultados obtenidos en el presente estudio han de interpretarse tomando en 
cuenta algunas limitaciones relevantes. En primer lugar, la muestra recolectada no es representativa de la población adulto joven ya que procede de un muestreo por conveniencia y su tamaño no es grande. En este sentido, los resultados pueden reflejar patrones que son más característicos de estudiantes universitarios con niveles socioeconómicos de medios a altos. En segundo lugar, el cuestionario que se utilizó para medir el apego no incluía una escala para medir el Apego Seguro. En estudios posteriores, por lo tanto, sería de interés evaluar los patrones correlacionales de este tipo de apego con los componentes de la calidad percibida de las relaciones de pareja.

Los hallazgos de esta investigación, de corroborarse en estudios futuros más representativos, tienen implicaciones prácticas relacionadas al abordaje terapéutico, tanto de las parejas como a nivel individual. Por un lado, la teoría del apego parece ser un elemento relevante a tomar en cuenta a la hora de diseñar y llevar a cabo las estrategias terapéuticas para tratar los problemas de relación de pareja, los cuales influyen especialmente sobre la salud social, emocional y física de las personas (Martínez et al., 2014). Además, tomando en consideración modelos que se desarrollan en base al apego, como la Terapia de Pareja Centrada en las Emociones y la Terapia Narrativa de Apego (Guzmán \& Contreras, 2012), se pueden crear intervenciones diferenciadas y prácticas que fomenten el desarrollo de la seguridad y el apoyo emocional entre los miembros de la pareja, así como la forma en que la persona se relaciona con los demás. Finalmente, al tomar en cuenta los Modelos Operativos Internos (Guzmán \& Contreras, 2012) y los estilos de apego adulto, los terapeutas pueden enriquecer su diagnóstico relacional 0 sistémico, y desarrollar un abordaje holístico que trabaje los patrones de apego disfuncionales en las relaciones de los individuos.

\section{REFERENCIAS}

Alonso-Arbiol, I., Balluerka, N., \& Shaver, P. R. (2007). A Spanish version of the experiences in close relationships (ECR) adult attachment questionnaire. Personal Relationships, 14(1), 45-63. doi:10.1111/j.1475-6811.2006.00141.x

Amato, P. R., \& Previti, D. (2003). People's Reasons for Divorcing: Gender, Social Class, the Life Course, and Adjustment. Journal of Family Issues, 24(5), 602626. doi:10.1177/0192513X03254507

Amato, P. R. (2000). The consequences of divorce for adults and children. Journal of Marriage and Family, 62(4), 1269$1287 . \quad$ doi:10.1111/j.17413737.2000.01269.x

Auslander, B. A., Short, M. B., Succop, P. A., \& Rosenthal, S. L. (2009). Associations between parenting behaviors and adolescent romantic relationships. Journal of Adolescent Health, 45(1), 28-101. doi:10.1016/j.jadohealth.2008.12.014

Bowlby, J. (1969). Attachment and loss: Vol. 1. Attachment. New York: Basic Books.

Bowlby, J. (1973). Attachment and loss: Vol. 2. Separation: Anxiety and anger. New York: Basic Books. 
Bowlby, J. (1980). Attachment and loss: Vol. 3. Loss. New York: Basic Books.

Brennan, K. A., \& Shaver, P. R. (1995). Dimensions of adult attachment, affect regulation, and romantic relationship functioning. Personality and Social Psychology Bulletin, 21(3), 267-283. doi: 10.1177/0146167295213008

Brennan, K. A., Clark, C. L., \& Shaver, P. R. (1998). Self-report measurement of adult attachment: An integrative overview. In J. A. Simpson \& W. S. Rholes (Eds.), Attachment theory and close relationships (pp. 46-76). New York: Guilford Press.

Bumpass, L.L., Martin, T.C., \& Sweet, J.A. (1991). The impact of family background and early marital factors on marital disruption. Journal of Family Issues, $\quad 12(1), \quad 22-42$. doi:10.1177/019251391012001003

Campbell, L., Simpson, J. A., Boldry, J., \& Kashy, D. A. (2005). Perceptions of conflict and support in romantic relationships: The role of attachment anxiety. Journal of Personality and Social Psychology, 88(3), 510-531. doi:10.1037/0022-3514.88.3.510

Cohen, J. (1992). A power primer. Psychological Bulletin, 112(1), 155-159. http://dx.doi.org/10.1037/00332909.112.1.155

Collins, N. L., \& Feeney, B. C. (2000). A safe haven: An attachment theory perspective on support seeking and caregiving in intimate relationships. Journal of Personality and Social Psychology, 78(6), 1053-1073. doi:10.1037/0022-3514.78.6.1053

Collins, N. L., \& Read, S. J. (1990). Adult attachment, working models, and relationship quality in dating couples. Journal of Personality and Social Psychology, 58(4), 644-663. doi: 10.1037/0022-3514.58.4.644

Collins, W. A., Welsh, D. P. \& Furman W. (2009). Adolescent Romantic Relationships. The annual review of psychology, 60, 631-631. doi:10.1146/annurev.psych.60.110707. 163459

Duemmeler, S. L. \& Kobak, R. (2001). The development of commitment and attachment in dating relationships: attachment security as relationship construct. Journal of Adolescence, 24(3), $\quad$ 401-415. doi:10.1006/jado.2001.0406

Feeney, B. C., \& Collins, N. L. (2001). Predictors of caregiving in adult Intimate relationships: An attachment theoretical perspective. Journal of Personality and Social Psychology, 80(6), 972-994. doi:10.1037/00223514.80 .6 .972

Fenney, J. A., \& Noller, P. (1990). Attachment style as a predictor of adult romantic relationships. Journal of Personality and Social Psychology, 58(2), 281-291. doi:10.1037/00223514.58.2.281

Feldman, S. S., Gowen, L. k., \& Fisher, L. (1998). Family relationships and gender as predictor of romantic intimacy in young adults: A longitudinal study. Journal of Research in Adolescence, 8, 263-286.

Fletcher, G. O., Simpson, J.A. \& Thomas, G. (2000). The measurement of perceived Relationship quality components: A confirmatory Factor analytic Approach. Personality and Social Psychology Bulletin, 26(3), 340-354. doi:10.1177/0146167200265007

Fraley, R. C., \& Davis, K. E. (1997). Attachment formation and transfer in young adults' close friendships and romantic relationships. Personal Relationships, $\quad 4, \quad 131-144$. doi:10.1111/j.14756811.1997.tb00135.x

George, D., \& Mallery, P. (2003). SPSS for Windows step by step: A simple guide and reference. 11.0 update (4th ed.). Boston: Allyn \& Bacon.

Gómez-Zapiain, J., Ortiz Barón, M. J., y Gómez Lope, J. (2012). Capacidad para aportar y solicitar apoyo emocional en las relaciones de pareja 
en relación con los perfiles de apego. Anales de Psicología, 28(1), 302-312.

Gottman, J. M. \& Levenson, R. W. (1999). What predicts change in marital interaction over time? A study of alternative models. Family Process, 38(2), 143-158. doi:10.1111/j.15455300.1999.00143.x

Griffin, D., \& Bartholomew, K. (1994b). Models of the self and other: Fundamental dimensions underlying measures of adult attachment. Journal of Personality and Social Psychology, $67(3)$, 430-445. doi:10.1037/00223514.67.3.430

Guzmán, M. \& Contreras, P. (2012). Estilos de apego en relaciones de pareja y su asociación con la satisfacción marital. Psykhe, 27(1), 69-82. doi:10.4067/S071822282012000100005

Hazan, C., \& Shaver, P. (1987) Romantic Love conceptualized as an attachment process. Journal of Personality and Social Psychology, 52(3), 511-524. doi:10.1037/0022-3514.52.3.511

Heffernan, M. E., Fraley, R. C., Vicary, A. M., Brumbaugh, C. C. (2012). Attachment features and functions in adult romantic relationships. Journal of Social and Personal Relationships, 29(5), 671-693. doi:10.1177/0265407512443435

Kachadourian, L. K., Fincham, F., \& Davila, J. (2004). The tendency to forgive in dating and married couples: The role of attachment and relationship satisfaction. Personal Relationships, 11, 373-393. doi:10.1111/j.14756811.2004.00088.x

Karney, B. R. \& Bradbury, T. N. (1995). The longitudinal course of marital quality and stability: A review of theory, method, and research. Psychological Bulletin, 118(1), 3-34. doi:10.1037/0033-2909.118.1.3

Kirkpatrick L. A., \& Davis K. E. (1994). Attachment style, gender, and relationship stability: A longitudinal analysis. Journal of Personality and Social Psychology, 66(3), 502-512. http://dx.doi.org/10.1037/0022-

3514.66.3.502

Leonard, K. E. \& Roberts, L. J. (1998). Marital Aggression, quality, and stability in the first year of marriage: findings from the Buffalo Newlywed Study. En T. N. Bradbury (Ed.), The developmental course of marital dysfunction. Cambridge, England: Cambridge University Press.

Martínez, J. L., Fuertes, A., Orgaz, B., Vicario, I. \& González, E. (2014). Vínculos afectivos en la infancia y calidad en las relaciones de pareja de jóvenes adultos: el efecto mediador del apego actual. Anales de psicología, 30(1), 211-220. doi:10.6018/analesps.30.1.135051

Maureira Cid, F. (2011). Los cuatro componentes de la relación de pareja. Revista electrónica de Psicología Iztacala, 14(1), 321-332.

Mikulincer, M., Florian, V., Cowan, P., \& Cowan, C. (2002). Attachment security in couple relationships: A systemic model and its implications for family dynamics. Family Process, 41(3), 405434. doi: 10.1111/j.15455300.2002.41309.x

Mikulincer, M., \& Shaver, P. (2007). Attachment in adulthood: Structure, dynamics, and change. New York: Guilford Press.

Mikulincer, M., Shaver, P. R., \& Pereg, D. (2003). Attachment theory and affect regulation: The dynamics, development, and cognitive consequences of attachment-related strategies. Motivation and Emotion, 27(2), 77-102. doi:10.1023/A:1024515519160

Molero, F., Shaver, P., Ferrer, E., Cuadrado, I., \& Alonso-Arbiol, I. (2010). Attachment insecurities and interpersonal processes in Spanish couples: A dyadic approach. Personal Relationships, 18(4), 617-629. doi:10.1111/j.1475-6811.2010.01325.x 
Ortiz Barón, M. J., Gómez Zapiain, J. y Apodaca, P. (2002). Apego y satisfacción afectivo sexual en la pareja. Psicothema, 2(14), 469-475.

Rivera, D., Cruz, C., \& Muñoz, C. (2011). Satisfacción en las relaciones de pareja en la adultez emergente: El rol del apego, la intimidad y la depresión. Terapia Psicológica, 29(1), 77-83.

Roisman, G.I., Holland, A., Fortuna, K., Fraley, R.C., Clausell, E., \& Clarke, A. (2007). The Adult Attachment Interview and self-reports of attachment style: An empirical rapprochement. Journal of Personality and Social Psychology, 92(4), 678-697. doi:10.1037/00223514.92.4.678

Simpson, J. A. (1990). Influence of attachment styles on romantic relationships. Journal of personality and social psychology, 59(5), 971-980. doi:10.1037/0022-3514.59.5.971

Tucker, J. S., \& Anders, S. L. (1999). Attachment style, interpersonal perception accuracy, and relationship satisfaction in dating couples. Personality and Social Psychology Bulletin, 43, 403-412. doi:10.1177/0146167299025004001

White, L. K. (1990). Determinants of divorce: A review of research in the eighties. Journal of Marriage and the Family, 52(4), 904-912.

Yárnoz-Yaben, S., Alonso-Arbiol, I., Plazaola, M., \& Sainz De Murieta, L. (2001). Apego en adultos y percepción de los otros. Anales de Psicología, 17(2), 159-170.

\section{APÉNDICE.}

Ítems Traducidos al español del Perceived Relationship Quality Components (PRQC).

\begin{tabular}{ll}
\hline Escala & Enunciado del Ítem \\
\hline Satisfacción & 1. ¿Qué tan satisfecho estás con tu relación? \\
& 2. ¿Qué tan contento estás con tu relación? \\
3. ¿Qué tan feliz estás con tu relación? & 4. ¿Qué tan comprometido estás con tu relación? \\
Compromiso & 5. ¿Qué tan dedicado estás a tu relación? \\
& 6. ¿Qué tan devoto eres a tu relación? \\
7. ¿Qué tan íntima es tu relación? \\
8. ¿Qué tan cercana es tu relación? \\
9. ¿Qué tan conectado estás a tu relación? \\
10. ¿Qué tanto confías en tu pareja? \\
11. ¿Qué tanto puedes contar con tu pareja? \\
12. ¿Qué tan confiable es tu pareja? \\
13. ¿Qué tan apasionada es tu relación? \\
14. ¿Qué tan lujuriosa es tu relación? \\
15. ¿Qué tan intensa a nivel sexual es tu relación? \\
16. ¿Qué tanto amas a tu pareja? \\
17. ¿Qué tanto adoras a tu pareja? \\
18. ¿Qué tanto aprecias a tu pareja?
\end{tabular}

